\title{
A Gold Nanoparticle Platform for Protein-Protein Interactions and Drug \\ Discovery
}

Andrew B. Thompson, ${ }^{\dagger}$ Amy K. Calhoun, ${ }^{\dagger}$ Benoit J. Smagghe, ${ }^{\dagger}$ Maria D. Stevens, ${ }^{\dagger, *}$ Mark T. Wotkowicz, ${ }^{\dagger}$ Vasilios M. Hatziioannou, ${ }^{\dagger}$ and Cynthia Bamdad ${ }^{\dagger}, *$

${ }^{\dagger}$ Minerva Biotechnologies, 40 Bear Hill Road, Waltham, MA 02451, USA.

Northeastern University, Boston, MA 02115, USA

Author Information.

Corresponding Author:

*Email: cbamdad@minervabio.com 


\section{Supplementary Results}

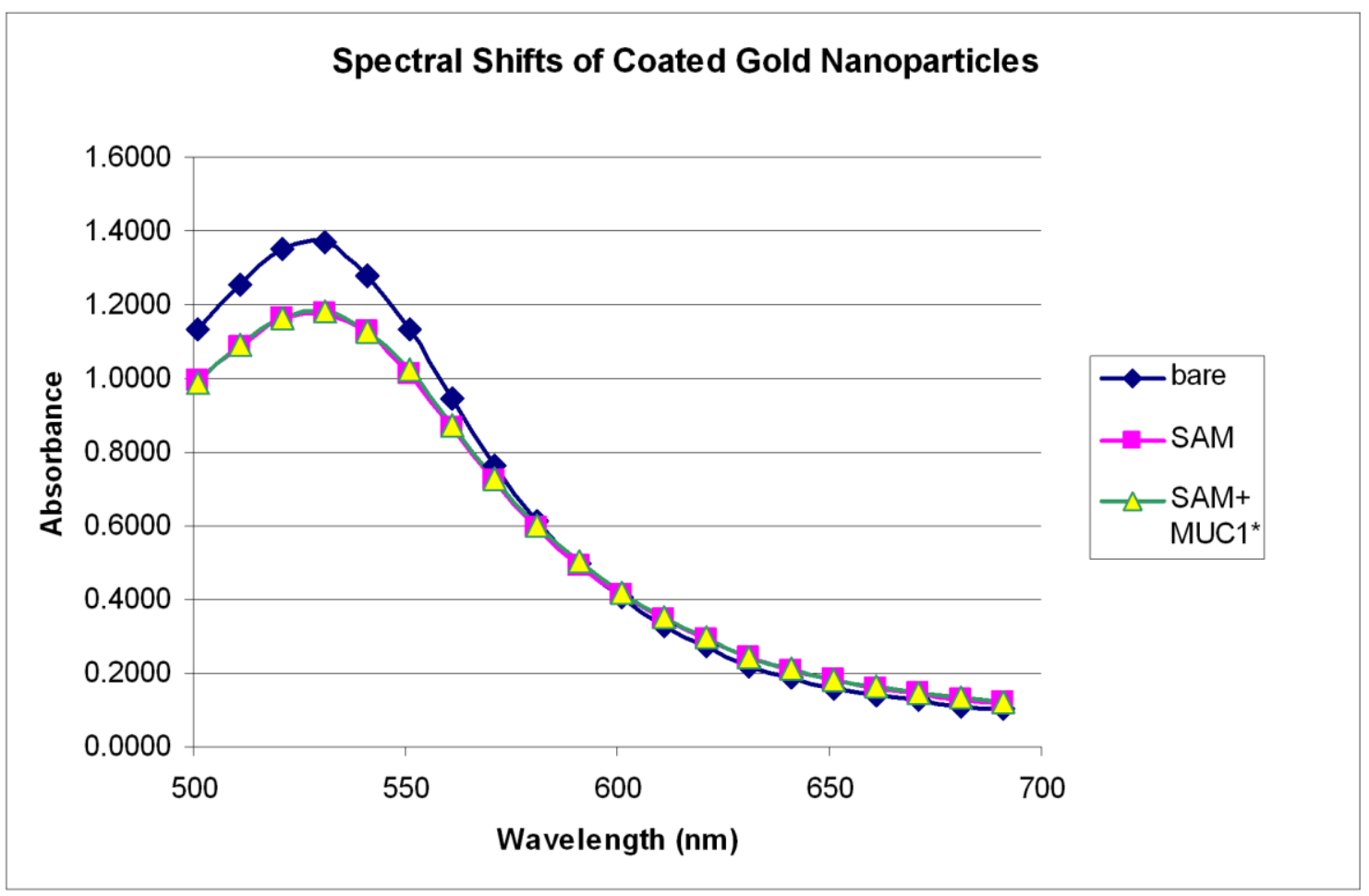

\section{Supplementary Figure 1. Absorbance of AuNP solutions with varying surface}

derivitization. The spectral absorbance from 500-700 nm was measured for bare nanoparticles, nanoparticles coated with an NTA-SAM, and nanoparticles coated with an NTA-SAM and loaded with MUC1* peptide. A slight decrease in absorbance from 500-560 nm was seen after coating with the NTA-SAM, but not from loading MUC1* peptide. No difference was measured between NTA-SAM coated AuNPs, and NTA-SAM coated AuNPs loaded with MUC1* peptide.

\section{Supplementary Discussion}

Aggregation analysis. Many quasiempirical methods for measuring aggregation of nanoparticles have been investigated. Most rely on an integration parameter that measures the area under the absorbance curve, usually between $600 \mathrm{~nm}$ and $800 \mathrm{~nm} .{ }^{1}$ While broadly useful, there is a drawback to this analysis in that it cannot account for precipitation of the nanoparticles. It is obvious to any observer that a nanoparticle solution that has precipitated is more aggregated than a nanoparticle solution that remains in suspension (for reference see Figure 4, part a). However, we observed that as growing colloidal aggregates begin to precipitate from solution, the overall absorbance of the solution decreases at all wavelengths. As precipitation increases, 
and absorbance necessarily decreases, the aggregation parameter will decrease as well, leading to a faulty impression that the suspension is un-aggregating. Thus, measuring aggregation by integrating the absorbance curve is only accurate for suspensions of colloidal particles that have not begun to precipitate. In our experiments, we needed to convey relative aggregation between conditions that contained precipitated aggregates and solutions that did not. It was observed that the change in absorbance at $521 \mathrm{~nm}$ continued to decrease throughout aggregation and eventual precipitation. We therefore chose to use this observation as the basis for measuring and calculating relative percent aggregation between samples. $\Delta \mathrm{A}_{521}$ was calculated as the change in absorbance at $521 \mathrm{~nm}$ between time $t$ and initial absorbance at $t_{0}$ (equation 1).

$$
\Delta A_{521}^{t}=A_{521}^{t}-A_{521}^{t_{0}}
$$

We could not define maximal aggregation, or total precipitation, as having an absorbance of 0 because the presence of the precipitates in the microplate well causes some absorbance. Therefore, our aggregation parameter, K, was calculated as a percent of maximal aggregation, whose value was determined experimentally by a completely precipitated sample after $24 \mathrm{hr}$ incubation at room temperature. Therefore, we defined this time point, for the sake of argument, as $t_{\infty}$ (equation 2).

$$
\mathrm{K}=\frac{\Delta A_{521}^{t}}{A_{521}^{t_{\infty}}-A_{521}^{t_{0}}} * 100
$$

\section{Supplementary Methods}

Gold Nanoparticle Synthesis. All glassware was soaked in aqua regia (Caution: Auqa Regia is extremely corrosive, use only in a fume hood equipped for corrosive vapors) for at least 2 hours prior to synthesis. Glassware was then rinsed repeatedly with Type I grade $\mathrm{H}_{2} \mathrm{O}$ (Ricca Chemical). $\mathrm{HAuCl}_{4} \cdot 3 \mathrm{H}_{2} \mathrm{O}(15.0 \mathrm{mg}, 38.1 \mu \mathrm{mol})$ was weighed into a clean, $500 \mathrm{~mL}$ glass round bottom flask. $150 \mathrm{~mL}$ of type $\mathrm{I} \mathrm{H}_{2} \mathrm{O}$ was then added, and the flask was placed in a heating mantle. With vigorous magnetic stirring, the solution was brought to a boil and a solution of $1.00 \%$ sodium citrate $(28.0 \mathrm{~mL}, 952 \mu \mathrm{mol}, 25$ equiv $)$ was added all at once. The solution immediately changed from a transparent, pale yellow to completely clear and colorless. Over the next 25 minutes, the solution slowly became steadily pinker, finally turning to a deep ruby red. Imaging of nanoparticles was performed by transmission electron microscopy on a JEOL 1200ex 
(Harvard Medical School) and images analyzed by ImageJ (available for free at: http://rsbweb.nih.gov/ij/) to determine nanoparticle diameter.

\section{Dynamic light scattering (DLS) measurements.}

The hydrodynamic radius ( $\mathrm{Rh}$ ) of AuNPs ( $15 \mathrm{~nm}$ to $30 \mathrm{~nm}$ ) was measured using a Viscotek 802 dynamic light scattering instrument (Viscotek, Houston, TX) at $20^{\circ} \mathrm{C}$. Each DLS experiment consisted of 60 runs of 5 seconds each. OmniSIZE 3.0 software was used to calculate the hydrodynamic radius of the particles based on the correlation function obtained from the DLS measurement.

Bare and NTA-Ni-SAM coated AuNPs loaded or not with the MUC1* peptide were diluted (20 times) into PBS pH 7.4 containing $0.01 \%$ Tween 20 and filtered with a $0.2 \mu$ m nylon syringe filter (PALL,Port Washington, NY), before the experiment.

For aggregation measurement, $50 \mu \mathrm{L}$ of non-diluted NTA-Ni-SAM coated AuNPs loaded with MUC1* peptide were incubated with $50 \mu$ l of anti MUC1* antibody solution $(10 \mu \mathrm{g} / \mathrm{mL}$, 33.3nM final concentration) and incubated for $30 \mathrm{~min}$. The samples were diluted 10 times before the DLS experiment.

Nanoparticle Salt Stress Assay. Nanoparticle batches of known average diameters (13.0, 14.9, 17.3, 19.2, 25.1, $31.1 \mathrm{~nm}$ ) were split into 3 lots. The first lot was coated with an NTASAM, the second lot was unmodified, and $0.01 \%$ Tween 20 was added in the third lot. Stock solutions of $\mathrm{NaCl}$ were prepared at concentrations of $4000 \mathrm{mM}, 3000 \mathrm{mM}, 2000 \mathrm{mM}, 1600 \mathrm{mM}$, $1200 \mathrm{mM}, 800 \mathrm{mM}, 400 \mathrm{mM}$, and $200 \mathrm{mM}$. In a 96-well microplate, $50 \mathrm{uL}$ of nanoparticle solution were mixed with $50 \mathrm{uL}$ of salt solution. Plates for this assay and for all the following were photographed over time to follow progress with an Olympus SP-350 digital camera.

Nanoparticles pH Stress Assay. $25 \mathrm{~nm}$ diameter AuNps were split into two batches, one was coated with a NTA-SAM and the other remained unmodified. Each batch was then split into 6 lots and concentrated by centrifugation at 14,000 rpm for 10 minutes. The supernatant was removed. The nanoparticles pellet were then resuspended into the following 6 buffers: $10 \mathrm{mM}$ Phosphoric Acid/Phosphate buffer $\mathrm{pH}$ 2.0, $10 \mathrm{mM}$ Citrate buffer $\mathrm{pH}$ 3.0, $10 \mathrm{mM}$ Citrate $\mathrm{pH}$ 5.0, $10 \mathrm{mM}$ Phosphate buffer $\mathrm{pH}$ 7.0, $10 \mathrm{mM}$ Carbonate buffer $\mathrm{pH} 9.0$ and $10 \mathrm{mM}$ Carbonate buffer 
$\mathrm{pH}$ 10.0. The nanoparticles were incubated for $1 \mathrm{~h}$ at room temperature and subjected to a salt stress assay as described above.

Peptide and Protein Attachment. $25 \mathrm{~nm}$ AuNPs were split into two batches, one of which was coated with a NTA-SAM and the other remained unmodified. To both batches of gold nanoparticles, a solution of $\mathrm{NiSO}_{4}$ was then added to a concentration of $0.001 \%$. The gold nanoparticles were incubated at room temperature for 5 minutes, and then centrifuged at 14,000 rpm for 10 minutes. The supernatants were removed and discarded, and the pellets were resuspended in $10 \mathrm{mM}$ phosphate buffer $\mathrm{pH}$ 7.4, $140 \mathrm{mM} \mathrm{NaCl}, 0.01 \%$ Tween 20. Each batch was then split and loaded with either of the following peptides (JPT Peptide Technologies, Berlin: 1) MUC1* peptide:

(GTINVHDVETQFNQYKTEAASRYNLTISDVSVSDVPFPFSAQSGAHHHHHH), 2) irrelevant GSD peptide: (GGDVGDSGGDVGDSGGDVGDSHHHHHH) or 3) irrelevant RDG peptide: (HHHHHH-SSSSGSSSSGSSSSGGRGDSGRGDS). Histidine-tagged SNAP25 and Syntaxin1a (Abcam, Cambridge, MA) were also used.

Antibody-induced Agglomeration Assay \#1 (Antibody specificity). Bare AuNPs and NTA-Ni-SAM coated AuNPs $(25 \mathrm{~nm})$ were separated in 3 lots and loaded with either $500 \mathrm{nM}$ MUC1* peptide, $250 \mathrm{nM} \mathrm{MUC1*}$ peptide or no peptide. The samples were incubated at room temperature for 10 minutes. $50 \mathrm{uL}$ from each lot were then plated into 5 wells of a single row of a 96 well plate, forming two 3x5 grids. Buffer and antibodies were added to a final volume of $100 \mathrm{uL}$. Anti-GFP antibody (Santa Cruz Biotechnology) and anti-MUC1* antibody (Minerva Biotechnologies, Waltham, MA) were added to final concentrations of $0,10 \mu \mathrm{g} / \mathrm{mL}$, and 20 $\mu \mathrm{g} / \mathrm{mL}$. Photos were taken over time to track progress.

Antibody-induced Agglomeration Assay \#2 (Peptide specificity). Two batches of NTANi-SAM coated AuNPs (25 nm) were separately loaded with $250 \mathrm{nM}$ of MUC1* peptide or 250 $\mathrm{nM}$ of the irrelevant GSD peptide. $50 \mathrm{uL}$ from each batch were added into 6 wells of a 96-well microplate, forming a 2x6 grid. Anti-MUC1* antibody (Minerva Biotechnologies, Waltham, $\mathrm{MA}^{28}$ ) was then added to each batch to final concentrations of $0,0.75,1.5,3.0,7.5$, and 15 $\mu \mathrm{g} / \mathrm{mL}$. Wells were thoroughly mixed, and photos taken over time to track progress. 
SNAP25 and Syntaxin1a binding Assay. Two batches of NTA-Ni-SAM coated gold nanoparticles were loaded with either histidine-tagged SNAP25 or histidine-tagged Syntaxin1a (Abcam, Cambridge, MA) to a final concentration of $1 \mu \mathrm{M}$ using the same method as described above. $150 \mu \mathrm{L}$ of these two batches were placed in individual wells of a 96 well plate, with an empty well between them. The plate was allowed to incubate at room temperature for $10 \mathrm{~min}$. After $10 \mathrm{~min}, 50 \mu \mathrm{L}$ from each of the two wells were mixed together in the middle well. The plate was again allowed to incubate at room temperature for $10 \mathrm{~min}$ and photographs were taken at indicated time points.

Nanoparticle Size Sensitivity Assay. NTA-Ni-SAM coated AuNPs of sizes 15, 20, 25, 30, \& $35 \mathrm{~nm}$ were loaded with MUC1* $(10 \mu \mathrm{M})$, incubated at rt for 10 minutes, then centrifuged at $14,000 \mathrm{rpm}$ at $4^{\circ} \mathrm{C}$. Supernatents were removed and discarded, and AuNP pellets were

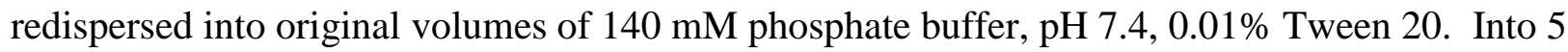
rows of a 96 well microplate, $50 \mu \mathrm{L}$ of colloid of each size batch was pipetted into 9 wells per row respectively, forming a $5 \times 9$ grid. Into each well was pipetted $50 \mu \mathrm{L}$ of $140 \mathrm{mM}$ phosphate buffer, $\mathrm{pH} 7.4,0.01 \%$ Tween 20 that contained the appropriate amount of $\alpha$-MUC1* antibody. Final concentrations of antibody tested on each size batch of nanoparticles were $0,0.67,3.33$, 6.67, 10.0, 13.3, 20.0, \& $33.3 \mathrm{nM} \alpha-\mathrm{MUC1} *$. Spectral measurements were taken on a Tecan Spectra Rainbow every 5 minutes covering a range from 501 to $691 \mathrm{~nm}$, incrementing every 10 nm. A photograph was taken at 2 hours.

Nanoparticles Temperature Stress Assay. NTA-Ni-SAM coated AuNPs (25 nm) were split into 4 sets of 5 batches. Each set was then maintained at ambient temperature, $37{ }^{\circ} \mathrm{C}$, $55^{\circ} \mathrm{C}$, or $80^{\circ} \mathrm{C}$. A sample from each temperature set was removed after: $30 \mathrm{~min}, 1 \mathrm{~h}, 2 \mathrm{~h}, 4 \mathrm{~h}$, and $6 \mathrm{~h}$. After cooling to room temperature, each batch was loaded with $\mathrm{NiSO}_{4}$ and $\mathrm{MUC1}$ * peptide $(500 \mathrm{nM})$ as described above and split into two lots. $50 \mathrm{uL}$ from each lot were mixed with buffer and either anti-MUC1* antibody (Minerva Biotechnologies, Waltham, MA) or antiGFP antibody (Santa Cruz biotechnology) to a final volume of $100 \mathrm{uL}$ per well and final concentration of $10 \mu \mathrm{g} / \mathrm{mL}$. Photos were taken after 30 minutes and a composite image was generated using Microsoft Powerpoint. 
Nanoparticles Freeze-thaw and Lyophilization Assay. A set of bare AuNPs containing $0.01 \%$ Tween 20 and a set of NTA-SAM coated AuNPs were split into 3 batches each. Batches were independently kept at $4{ }^{\circ} \mathrm{C}$, frozen at $-70{ }^{\circ} \mathrm{C}$ for $2 \mathrm{~h}$ then thawed, or freeze-dried then reconstituted. All batches were loaded with $\mathrm{NiSO}_{4}$ and $\mathrm{MUC1}{ }^{*}$ peptide as described above, to a final concentration of $250 \mathrm{nM}$. Each batch was then subjected to antibody cross-linking with either anti-MUC1* antibody (Minerva Biotechnologies, Waltham, MA), anti-MUC1* Fab fragment, or irrelevant anti-GFP antibody (Santa Cruz Biotechnology). A photograph was taken at $60 \mathrm{~min}$.

Lyophilization of Peptide Loaded Gold Nanoparticles. A set of NTA-Ni-SAM coated AuNPs was loaded with MUC1* peptide as described above to a final concentration of $250 \mathrm{nM}$. This batch was then frozen, lyophilized, and then reconstituted. The set was split into two batches that were subjected to antibody-crosslinking with either either anti-MUC1* antibody (Minerva Biotechnologies, Waltham, MA) or irrelevant anti-GFP antibody (Santa Cruz Biotechnology) to a final concentration of $10 \mu \mathrm{g} / \mathrm{mL}$. A photograph was taken after $60 \mathrm{~min}$.

High Throughput Drug Screening. $2.5 \mathrm{~mL}$ of NTA-Ni-SAM coated AuNPs were incubated with a $10 \mu \mathrm{M} \mathrm{MUC1*}$ peptide solution, pelleted, then rinsed. Additionally, $200 \mu \mathrm{L}$ of NTA-Ni-SAM coated AuNPs were loaded with a $10 \mu \mathrm{M}$ irrelevant RGD peptide solution, pelleted, then rinsed. $65 \mu \mathrm{L}$ of freshly prepared T47D (ATCC) cell lysate was added to each well of a 96 well plate, excluding the final column. Into the first column of wells, $5 \mu \mathrm{L}$ of PBS was mixed into the lysate. Into the remaining wells, $5 \mu \mathrm{L}$ of compound $(1 \mathrm{mg} / \mathrm{mL}$ in DMSO) from a drug-screening library (Comgenex) were mixed into the lysate and incubated at room temperature for 10 minutes. As positive and negative controls, $30 \mu \mathrm{L}$ of the MUC1*-loaded gold nanoparticles were added to the 4 first wells of column 1 and $30 \mu \mathrm{L}$ of the RGD-loaded gold nanoparticles were added to the last 4 wells, and wells were mixed thoroughly. Pictures were taken over time. 


\section{REFERENCES}

(1) Weisbecker, C. S.; Merritt, M. V.; Whitesides, G. M. Langmuir 2005, 12, 3763-3772. 